\title{
Endodontic management of single rooted immature mandibular second molar with single canal using MTA and platelet-rich fibrin membrane barrier: A case report.
}

\author{
Ambica Kathuria ${ }^{1}$, Sarika Chaudhry ${ }^{2}$, Sangeeta Talwar ${ }^{3}$, Mahesh Verma ${ }^{4}$ \\ ${ }^{1}$ MDS . Senior Research Associate, Department of Conservative dentistry \& Endodontics, Maulana Azad Institute of Dental \\ Sciences, New Delhi- 110002, India. \\ ${ }^{2}$ MDS . Associate Professor, Department of Conservative dentistry \& Endodontics, Maulana Azad Institute of Dental Sciences, \\ New Delhi- 110002, India. \\ ${ }^{3}$ MDS . Professor \& Head of Department, Department of Conservative dentistry \& Endodontics, Maulana Azad Institute of \\ Dental Sciences, New Delhi- 110002, India. \\ ${ }^{4}$ MDS . Director- Principal, Department of Prosthodontics, Maulana Azad Institute of Dental Sciences, New Delhi- 110002, \\ India.
}

\author{
Correspondence: \\ Department of Conservative Dentistry and Endodontics \\ Maulana Azad Institute of Dental Sciences, \\ MAMC complx, BSZ marg, Delhi-110002. \\ Email :drambics@yahoo.com
}

\author{
Kathuria A, Chaudhry S, Talwar S, Verma M. Endodontic management of \\ single rooted immature mandibular second molar with single canal using \\ MTA and platelet-rich fibrin membrane barrier: A case report. J Clin Exp \\ Dent. 2011;3(5):e487-90. \\ http://www.medicinaoral.com/odo/volumenes/v3i5/jcedv3i5p487.pdf \\ Article Number: $50544 \quad$ http://www.medicinaoral.com/odo/indice.htm \\ (C) Medicina Oral S. L. C.I.F. B 96689336 - eISSN: 1989-5488 \\ eMail: jced@jced.es
}

\begin{abstract}
An immature tooth with pulpal necrosis and periapical pathology imposes a great difficulty to the endodontists. Endodontic treatment options for such teeth consist of conventional apexification procedure with and without apical barriers. Various materials have been tried as apical matrix in the past. This article demonstrates the newer concept of using platelet rich fibrin membrane as an apical matrix barrier for stabilization of MTA in root end apexification procedure. Platelet Rich Fibrin is an autologous fibrin matrix containing a large quantity of platelet and leukocyte cytokines, which enhance healing by release of growth factors. This case report represents successful management of a case of an immature mandibular second molar with single root and single c-shaped canal. The unusual canal morphology was diagnosed with the help of a CBCT scan. Faster periradicular healing and complete resolution of symptoms occurred with combined use of MTA and PRF membrane as an apical barrier.
\end{abstract}

Key words: Apexification; apical barrier; platelet rich fibrin (PRF); mineral trioxide (MTA) 


\section{Introduction}

A study by Weine et al reported that $1.3 \%$ of mandibular second molars have single canal configuration (1) This case report highlights the occurrence and management of single rooted mandibular second molar with open apex that required endodontic therapy.

MTA has been shown to be a very effective root filling material for sealing immature root canals with open apices that could otherwise impose technical challenges in obtaining adequate obturation. MTA has an ability to facilitate periradicular healing by inducing hard-tissue formation (2). But in some cases with wide open apices, adequate condensation of MTA is difficult to achieve as the material may get extruded beyond the apex. Therefore an apical matrix is used for the controlled placement of MTA to a desired level. Various biocompatible materials have been tried as apical matrix in the past. These include: tricalcium phosphate (3), collagen calcium phosphate (4)osteogenic protein-1, bone growth factor and oxidized cellulose (5) etc. "Modified matrix concept" for repair of perforation utilized resorbable collagen as a matrix followed by condensation of MTA (6).

Platelet-rich fibrin (PRF) developed in France by Choukroun and Dohan (7) represents a new step in the platelet gel therapeutic concept. PRF is a matrix of autologous fibrin, in which are embedded a large quantity of platelet and leukocyte cytokines during centrifugation. PRF obtained from Choukroun's technique is a strong fibrin membrane enriched with platelet and growth factors. The easily applied PRF membrane serves as a matrix to accelerate the healing of wound edges (8).

Therefore, present case report highlights the management of a single rooted mandibular second molar with immature apex and periapical radiolucency using PRF membrane matrix and MTA to promote periapical healing.

\section{Case Report}

A 15-year-old male patient complained of pain in relation to the left mandibular posterior region, especially after consuming hot drinks. Medical history was noncontributory. Intraoral examination revealed deep caries. The tooth was also tender on percussion. Intraoral periapical radiograph of the tooth revealed deep caries approximating the pulp with associated periapical pathology and open apex (Figure 1). Vitality test for heat and cold were negative. A detailed examination of the radiograph revealed the presence of a single root with a wide canal. Therefore c-shaped canal configuration was anticipated. To confirm this unusual morphology, it was decided to perform CBCT imaging of the tooth. An informed consent was obtained from the patient, and a multislice CBCT scan was performed. The involved tooth was focused, and the morphology was obtained in transverse, axial, and sagittal sections of $0.5-\mathrm{mm}$ thick-

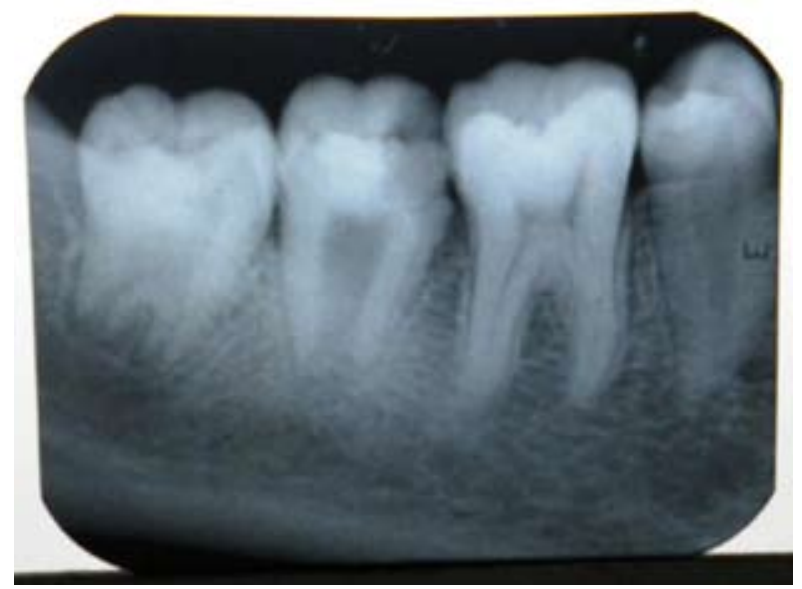

Fig. 1. Preoperative radiograph

ness. CBCT scan slices revealed single wide c-shaped canal with wide open apex.

Endodontic access opening was done under local anesthesia, and a periapical radiograph was taken to determine the working length. The root canal was lightly cleaned with a hand file under irrigation with $1.3 \% \mathrm{NaOCl}$. The root canal was then dried with sterile paper points. Calcium hydroxide was placed in the root canal, and the patient was recalled after one week. One week later, the tooth was again isolated under rubber dam, the calcium hydroxide dressing was removed by hand instrumentation, and irrigation was done with $1.3 \% \mathrm{NaOCl}$ and $17 \%$ liquid EDTA Smear Clear (SybronEndo, CA, USA). The root canal was then dried with sterile paper points.

It was decided to use PRF membrane in the formation of the artificial barrier. For this, approval was obtained from the ethical committee of Maulana Azad Institute of Dental Sciences, New Delhi, India. Also, informed consent was obtained from the patient after explaining the whole procedure. Patient's whole blood was drawn into $10 \mathrm{ml}$ glass coated plastic tubes using PRF collection kit without anticoagulant and immediately centrifuged in Process ${ }^{\circledR}$ centrifuge (PC-02, Process Ltd., Nice, France) at 3000rpm for 10 minutes. Three layers got formed in the tube: a base of Red Blood Corpuscles (RBCs), at the bottom, acellular plasma on the surface, and PRF clot in the middle. The fibrin clot was easily separated from the lower part of the centrifuged blood. (Figure 2) The PRF clot was gently pressed into a membrane with a sterile dry gauge (9).

The membrane was packed against the bone and was pushed beyond the apex into the bony space formed due to the periapical lesion to form a matrix for the placement of MTA.

A thick mixture of White Proroot MTA (Dentsply, Switzerland) was then prepared and applied to the apical portion of the canal using a small plugger and the butt end of sterile paper points and excess material was cleared from the walls. Moistened gauze was placed in the remainder 


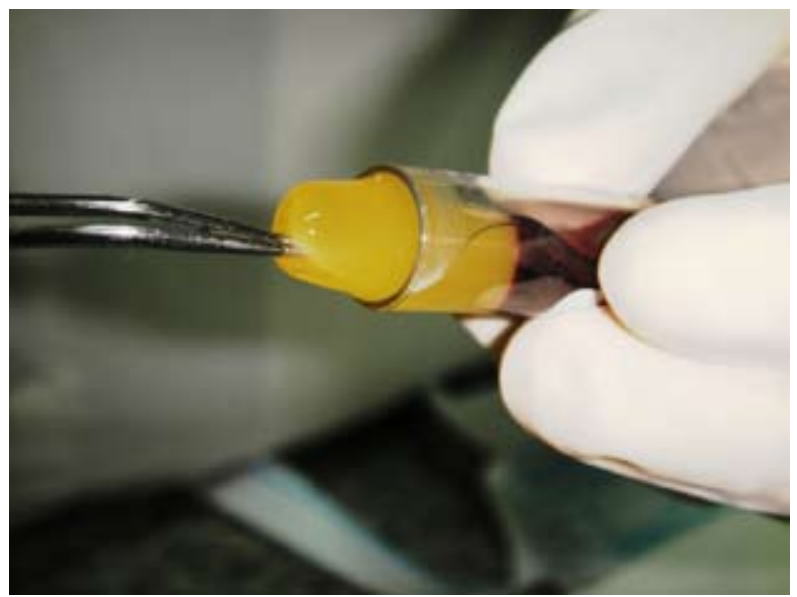

Fig. 2. Fibrin clot is gently grabbed with pliers to yield PRF membrane

of the canal and the access cavity sealed using Type-II restorative glass ionomer cement (Fuji, GC Corporation, Tokyo, Japan). In the next visit, Gutta percha backfill was performed using Obtura (Obtura/Spartan, Fenton, MO, USA), and the access cavity was sealed using composite resin. A radiograph confirmed the completion of the endodontic therapy.

The clinical follow-up at 18 months showed the patient functioning well with no reportable clinical symptoms and an absence of any sinus tract formation. The radiographic follow-up (Figure 3) showed complete healing of the periapical radiolucency and regeneration of the osseous periradicular tissues.

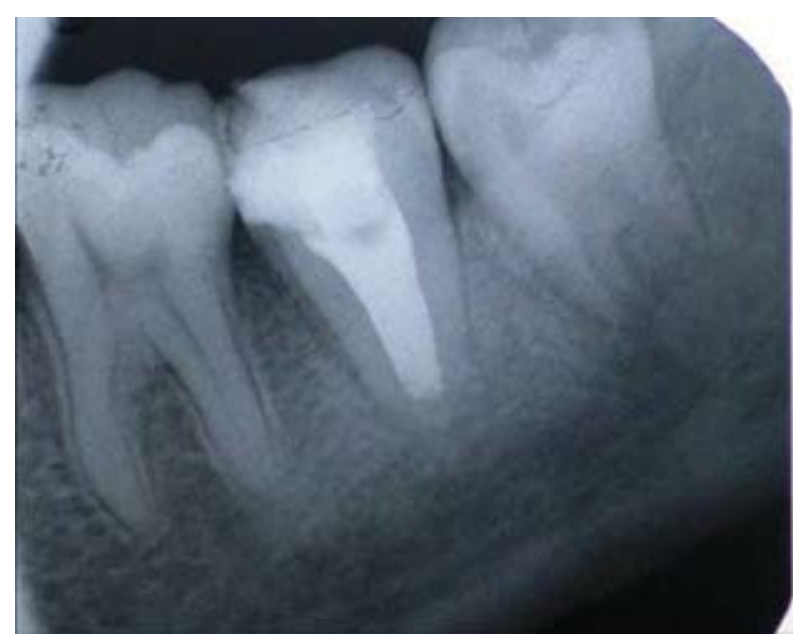

Fig. 3. periradicular healing evident after 18 months

\section{Discussion}

The major problem in cases of a wide open apex is the need to limit the material, thus avoiding the extrusion of a large amount of material into the periapical tissue. Using a matrix avoids the extrusion of the material into the periodontal tissues, reduces leakage in the sealing material and allows favorable response of the periodontal tissues. The apical barrier technique utilizing calcium sulfate or a combination of calcium sulfate and collagen in a powdered form has been performed in the past. Various materials have been used for formation of apical barrier during apexification. This case report has introduced a new concept of using PRF as an apical matrix membrane. PRF is a matrix of autologous fibrin, in which are embedded a large quantity of platelet and leukocyte cytokines during centrifugation (7). The intrinsic incorporation of cytokines within the fibrin mesh allows for their progressive release over time (7-10 days), as the network of fibrin disintegrates (8). According to Simonpieri A et al (10), the use of this platelet and immune concentrate offers the following 4 advantages: First, the fibrin clot plays an important mechanical role, with the PRF membrane maintaining and protecting the grafted biomaterials and PRF fragments serving as biological connectors between bone particles. Second, the integration of this fibrin network into the regenerative site facilitates cellular migration, particularly for endothelial cells necessary for the neo-angiogenesis (8), vascularization and survival of the graft. Third, the platelet cytokines (PDGF, TGF- $\square$, IGF-1) are gradually released as the fibrin matrix is resorbed, thus creating a perpetual process of healing. Lastly, the presence of leukocytes and cytokines in the fibrin network can play a significant role in the self-regulation of inflammatory and infectious phenomena within the grafted material.

Studies have proved high affinity of osteoblasts to the PRF membrane that suggests its excellent osseous healing potential (11). Various case reports have described the use of PRF as a graft for ridge preservation procedures, to enhance healing of the extraction socket (12) and to obtain predictable esthetic root coverage in cases of gingival recession (13). In this case, similar osseous response was obtained that was confirmed by the radiographic bone fill. But, histological techniques could not be performed to identify the new tissue formed as it involved surgical intervention. This remains a limitation and further animal studies are required to demonstrate the osseous healing potential of PRF in such situations.

\section{Conclusion}

The combination of PRF membrane as a matrix and MTA can prove to be an effective alternative for creating artificial root-end barriers and to induce faster periapical healing with large periapical lesions. The precise mechanism of action of PRF is yet to be explored. PRF is a second generation platelet concentrate which is still under study and many more advancements in its clinical applications are expected in near future.

\section{Acknowledgement}

We would like to acknowledge Council for Scientific 
and Industrial Research (CSIR), New Delhi, India.

\section{References}

1. Weine FS, Pasiewicz RA, Rice RT. Canal configuration of the mandibular second molar using a clinically oriented in vitro method. J Endod. 1988;14:207-13.

2. Giuliani V, Baccetti T, Pace R, Pagavino G. The use of MTA in teeth with necrotic pulps and open apices. Dent Traumatol. 2002;18:217-21.

3. Coviello J, Brilliant JD. A preliminary clinical study on the use of tricalciumphosphate as an apical barrier. J Endod. 1979;5:6-13.

4. Nevins A, Finkelstein F, Laporta R, Borden BG. Induction of hard tissue into pulpless open-apex teeth using collagen-calcium phosphate gel. J Endod. 1978;4:76-81.

5. Dimashkieh MR. A method of using silver amalgam in routine endodontics, and its use in open apices. Br Dent J. 1975;138:298 300.

6. Bargholz C. Perforation repair with mineral trioxide aggregate: A modified matrix concept. Int Endod J. 2005;38:59-69.

7. Dohan DM, Chokroun J, Diss A, Dohan SL, Dohan AJ, Mouhyi J et al. Platelet-rich fibrin (PRF): a second generation platelet concentrate- part I: technological concept and evolution. Oral Surg Oral Med Oral Pathol Oral Radiol Endod. 2006;101:e37-44.

8. Simonpieri A, Del Corso M, Sammartino G, Dohan Ehrenfest DM The relevance of Choukroun's platelet-rich fibrin and metronidazole during complex maxillary rehabilitations using bone allograft. Part I: a new grafting protocol. Implant Dent. 2009;18:102-11.

9. Huang FM, Yang SF, Zhao JH, Chang YC. Platelet-rich fibrin increases proliferation and differentiation of human dental pulp cells. J Endod. 2010;36:1628-31.

10. Simonpieri A, Del Corso M, Sammartino G, Dohan Ehrenfest DM. The relevance of Choukroun's platelet-rich fibrin and metronidazole during complex maxillary rehabilitations using bone allograft. Part II: implant surgery, prosthodontics, and survival. Implant Dent. 2009;18:220-29.

11. Sanchez AR, Sheridan PJ, Kupp LI. Is platelet rich plasma the perfect enhancement factor? A current Review. Int J Oral Maxillofac Implants. 2003;18:93-103.

12. Simon BI, Zatcoff AL, Kong JJW, and O'Connell SM. Clinical and histological comparison of extraction socket healing following the use of autologous platelet-rich fibrin matrix (prfm) to ridge preservation procedures employing demineralized freeze dried bone allograft material and membrane. Open Dent J. 2009;3: 92-9.

13. Anilkumar K, Geetha A, Umasudhakar, Ramakrishnan T, Vijayalakshmi R, Pameela E. Platelet-rich-fibrin: A novel root coverage approach. J Indian Soc Periodontol. 2009;13:50-4 